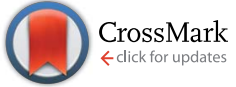

Cite this: RSC Adv., 2016, 6, 74765

Received 26th June 2016

Accepted 3rd August 2016

DOI: $10.1039 / c 6 r a 16486 b$

www.rsc.org/advances

\title{
Magnetic properties and giant reversible magnetocaloric effect in $\mathrm{GdCoC}_{2}$
}

\begin{abstract}
Lingjian Meng, ${ }^{\text {ab }}$ Chi Xu, ${ }^{c}$ Ye Yuan, ${ }^{c}$ Yang Qi, ${ }^{b}$ Shengqiang Zhou ${ }^{c}$ and Lingwei Li ${ }^{\star a b}$
The crystal structure, magnetic properties and magnetocaloric effect (MCE) of $\mathrm{GdCoC}_{2}$ have been studied. The compound crystallizes in an orthorhombic $\mathrm{CeNiC}_{2}$-type structure which belongs to the Amm2 space group. A giant reversible $\mathrm{MCE}$ is observed in $\mathrm{GdCoC}_{2}$ accompanied by a second-order paramagnetic to ferromagnetic (PM-FM) phase transition around the Curie temperature $\sim 15 \mathrm{~K}$. For the magnetic field change of $0-5 T$, the maximum values of the magnetic entropy change $\left(-\Delta S_{M}^{\max }\right)$, relative cooling power (RCP), and refrigerant capacity (RC) are $28.4 \mathrm{~J} \mathrm{~kg}^{-1} \mathrm{~K}^{-1}, 566 \mathrm{~J} \mathrm{~kg}^{-1}$ and $369 \mathrm{~J} \mathrm{~kg}^{-1}$, respectively. The present results indicate that $\mathrm{GdCoC}_{2}$ is a promising candidate for low temperature magnetic refrigeration.
\end{abstract}

\section{Introduction}

Materials with large/giant magnetocaloric effect (MCE) have gained more and more interest due to their applications for magnetic refrigeration. Compared with traditional gas refrigeration, magnetic refrigeration has significant advantages in conversion efficiency, low noise and environmental protection. ${ }^{1-5}$ The MCE manifests as $\Delta S_{\mathrm{M}}$ (isothermal magnetic entropy change) and $\Delta T_{\mathrm{ad}}$ (adiabatic temperature change). Finding special materials with a large MCE value is considered to be the most important work of magnetic refrigeration, since the MCE is an inherent characteristic of magnetic materials. In recent years, rare-earth based compounds have been widely investigated with respect to their MCE properties. ${ }^{6-19}$ And some of them exhibit large/giant MCE at 10-25 K, which is around the boiling point of hydrogen. ${ }^{6-15}$ Considering the problems in application and storage of liquid hydrogen at room temperature, these materials are eligible to be applied for magnetic refrigeration for hydrogen liquefaction.

In the present study, we have investigated the MCE in $\mathrm{GdCoC}_{2}$ which belongs to an existing ternary system $\mathrm{RETC}_{2}(\mathrm{RE}$ stands for heavy rare earth element and $\mathrm{T}$ stands for transition metal Co and Ni). Schafer et al. have reported that all the $\mathrm{RECoC}_{2}$ compounds order ferromagnetically, whereas, compounds of $\mathrm{RENiC}_{2}$ are antiferromagnetic. ${ }^{20-26} \mathrm{Up}$ to the present, only the $\mathrm{MCE}$ in $\mathrm{TbCoC}_{2}$ belonging to this series compounds has been reported and the maximum magnetic entropy change $\left(-\Delta S_{\mathrm{M}}^{\max }\right)$ is $15.3 \mathrm{~J} \mathrm{~kg}^{-1} \mathrm{~K}^{-1}$ for the magnetic field change of $0-5 \mathrm{~T}^{15}$ For the presently studied $\mathrm{GdCoC}_{2}$, the

${ }^{a}$ Key Laboratory of Electromagnetic Processing of Materials (Ministry of Education), Northeastern University, Shenyang 110819, China. E-mail: lingwei@epm.neu.edu.cn ${ }^{b}$ Institute of Materials Physics and Chemistry, School of Materials Science and Engineering, Northeastern University, Shenyang 110819, China

'Helmholtz-Zentrum Dresden-Rossendorf, Institute of Ion Beam Physics and Materials Research, Bautzner Landstrasse 400, 01328 Dresden, Germany values of $-\Delta S_{\mathrm{M}}^{\max }$ are $16.0 \mathrm{~J} \mathrm{~kg}^{-1} \mathrm{~K}^{-1}, 28.4 \mathrm{~J} \mathrm{~kg}^{-1} \mathrm{~K}^{-1}$, and $32.9 \mathrm{~J}$ $\mathrm{kg}^{-1} \mathrm{~K}^{-1}$ for magnetic field changes of $0-2 \mathrm{~T}, 0-5 \mathrm{~T}$ and $0-7 \mathrm{~T}$, respectively.

\section{Experimental details}

The polycrystalline $\mathrm{GdCoC}_{2}$ sample was synthesized by the method of arc-melting. First, stoichiometric amounts of highpurity components were weight and $3 \%$ extra carbon was added to compensate the loss during arc-melting. The sample was turned over and melted for four times under an argon atmosphere to ensure good homogeneity. Then the sample was annealed at $900{ }^{\circ} \mathrm{C}$ for six days in evacuated quartz tubes and rapidly quenched in ice water. The sample was characterized by X-ray powder diffraction (XRD) measurement by Rigaku D/MAX 2550 using $\mathrm{Cu} \mathrm{K} \alpha$ radiation. The magnetization measurements were done with a commercial superconducting quantum interference device (SQUID) based vibrating sample magnetometer (VSM) (Quantum Design Company, USA).

\section{Results and discussion}

The XRD pattern for $\mathrm{GdCoC}_{2}$ together with the Rietveld refinement profiles which were analyzed by MAUD software are shown in Fig. 1. The inset of Fig. 1 shows the crystal structure diagram obtained by using the CRYSTALMAKER software package. The factors of Rietveld refinement which were calculated by MAUD software are $R_{\mathrm{wp}}(\%)=8.99, R_{\mathrm{B}}(\%)=6.69, R_{\exp }$ $(\%)=12.0$. The results reveal that the sample is crystallized in single phase with orthorhombic $\mathrm{CeNiC}_{2}$-type structure (Amm2 space group) and no impurity phases can be detected. According to the Rietveld refinement profiles, the refined lattice parameters $a, b$, and $c$ are calculated by Bragg equation and found to be $3.621,4.506$, and $6.062 \AA$, respectively, which are close to the standard values. 


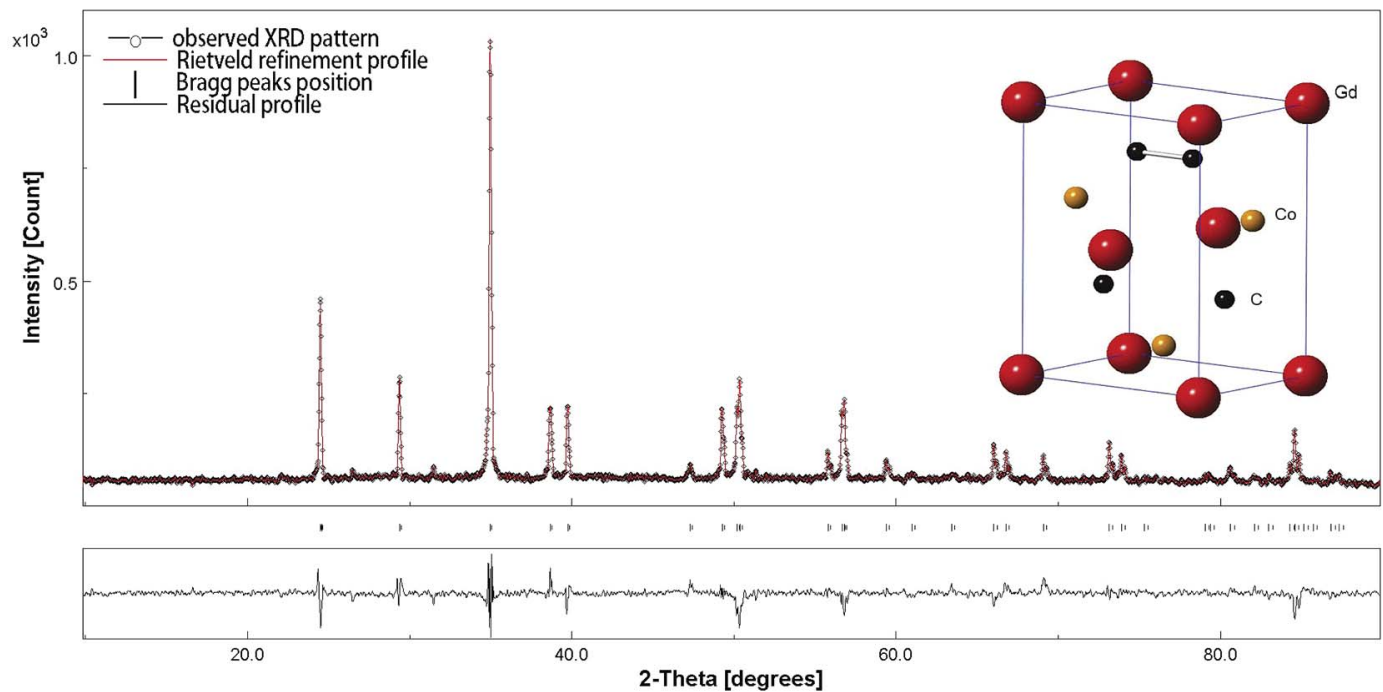

Fig. 1 XRD pattern for $\mathrm{GdCoC}_{2}$ together with the Rietveld refinement profiles, Bragg peak positions, Rietveld profiles. The inset shows the crystal structure diagram.

The zero field cooling (ZFC) and field cooling (FC) magnetization $(M)$ at magnetic field $(H)$ of $0.2 \mathrm{~T}$ is shown in the inset of Fig. 2 . A typical paramagnetic to ferromagnetic (PM-FM) phase transition can be observed at the Curie temperature $\left(T_{\mathrm{C}}\right) \sim 15 \mathrm{~K}$ (defined as the minimum value of $\mathrm{d} M / \mathrm{d} T$ curve). No obvious difference can be observed between ZFC and FC curves, which is a typical behavior for the second order phase transition materials. The temperature dependence of magnetization $(M)$ and the inverse susceptibility $(1 / \chi)$ under magnetic field $(H)$ of $1 \mathrm{~T}$ are shown in Fig. 2. At high temperature, the $1 / \chi-T$ curve shows Curie-Weiss behavior. The evaluated value of the effective magnetic moment (8.0 $\mu_{\mathrm{B}} /$ f.u.) for $\mathrm{GdCoC}_{2}$ is close to that of the free ion value of $\mathrm{Gd}^{3+}$ $\left(7.94 \mu_{\mathrm{B}}\right)$. A set of magnetization isotherm $(M-H)$ was measured at temperatures from $3 \mathrm{~K}$ to $45 \mathrm{~K}$ under magnetic field up to $7 \mathrm{~T}$, and several $M-H$ curves with increasing field are shown in Fig. 3. Among them, the hysteresis cycles at $3 \mathrm{~K}, 13 \mathrm{~K}, 15 \mathrm{~K}, 20 \mathrm{~K}$, and $30 \mathrm{~K}$

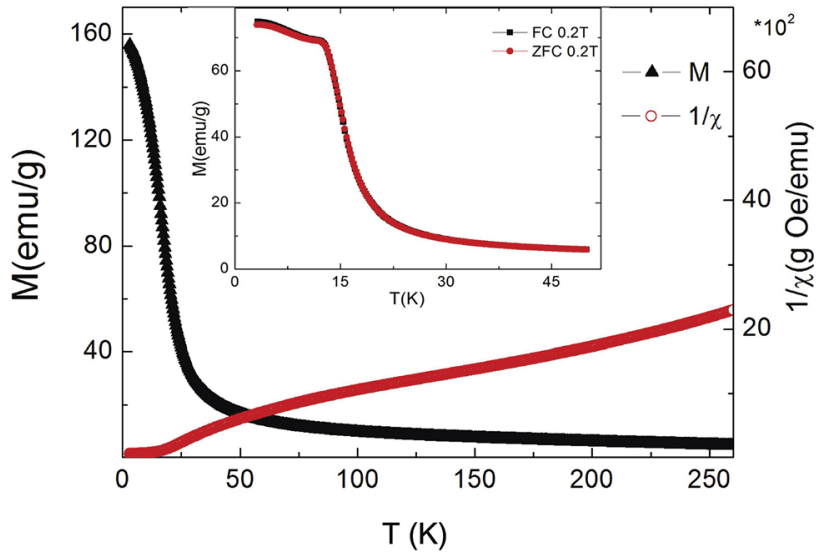

Fig. 2 Temperature dependence of magnetization (left scale) and the inverse susceptibility (right scale) for $\mathrm{GdCoC}_{2}$. The inset shows the temperature dependence of zero field cooling (ZFC) and field cooling (FC) magnetization $(M)$ under magnetic fields $(H)=0.2 \mathrm{~T}$. are shown in the figure as well. No obvious hysteresis is observed and the magnetization rapidly changes with varying temperature around $T_{\mathrm{C}}$, i.e. a large reversible MCE may appear around $15 \mathrm{~K}$. The inset of Fig. 3 shows the $M^{2}$ versus $H / M$ curves (also named as Arrot plot) from $3 \mathrm{~K}$ to $45 \mathrm{~K}$. Based on the Banerjee criterion, ${ }^{27}$ the present $\mathrm{GdCoC}_{2}$ undergoes a second-order magnetic phase transition since neither negative slope nor inflection can be observed in the whole temperature range.

The isothermal magnetic-entropy change $\Delta S_{\mathrm{M}}$ is obtained from magnetization isotherms by integrating the Maxwell relation

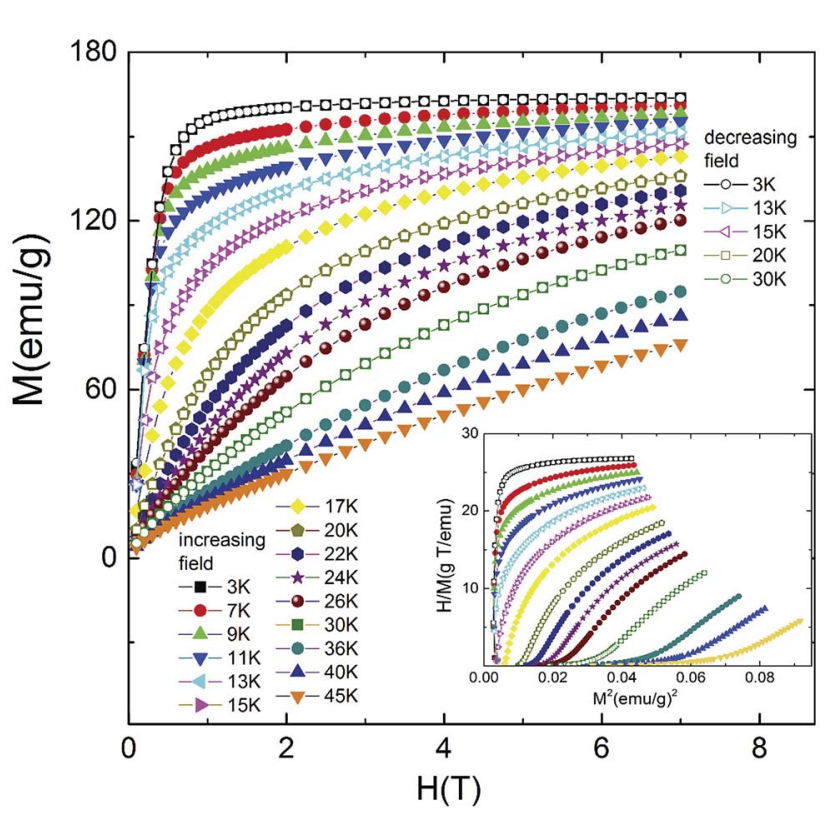

Fig. 3 Magnetic field $(H)$ dependence of the magnetization $(M)$ for $\mathrm{GdCoC}_{2}$ at different temperatures. The inset shows the Arrot plots curves $\left(M^{2}\right.$ versus $\left.H / M\right)$. 


$$
\begin{aligned}
\Delta S_{\mathrm{M}}(T, \Delta H)= & \int_{0}^{H_{\max }}\left(\frac{\partial M}{\partial T}\right)_{H} \mathrm{~d} H \approx \frac{1}{\Delta T} \int_{0}^{H_{\max }}\left[M\left(T_{i+1}, H\right)\right. \\
& \left.-M\left(T_{i}, H\right)\right] \mathrm{d} H,
\end{aligned}
$$

The temperature-dependent $-\Delta S_{\mathrm{M}}$ under magnetic field changes up to 0-7 $\mathrm{T}$ is shown in Fig. 4. $-\Delta S_{\mathrm{M}}$ increases with the increasing value of $\Delta H$. The maxima of $-\Delta S_{\mathrm{M}}$ appears around $17 \mathrm{~K}$, which is close to the paramagnetic to ferromagnetic (PM$\mathrm{FM})$ phase transition. The values of $-\Delta S_{\mathrm{M}}^{\max }$ are equal to $9.3 \mathrm{~J}$ $\mathrm{kg}^{-1} \mathrm{~K}^{-1}, 20.9 \mathrm{~J} \mathrm{~kg}^{-1} \mathrm{~K}^{-1}, 28.4 \mathrm{~J} \mathrm{~kg}^{-1} \mathrm{~K}^{-1}, 32.9 \mathrm{~J} \mathrm{~kg}^{-1} \mathrm{~K}^{-1}$ under the field changes of $0-1 \mathrm{~T}, 0-3 \mathrm{~T}, 0-5 \mathrm{~T}$ and $0-7 \mathrm{~T}$, respectively. These values are around two times of those for $\mathrm{TbCoC}_{2}$ and larger than those of most of recently reported giant MCE materials in the same temperature range, indicating that the presently studied $\mathrm{GdCoC}_{2}$ compound belongs to a class of giant MCE materials. The origin of giant $\mathrm{MCE}$ in $\mathrm{GdCoC}_{2}$ is probably related to the large saturation moment, especially under low magnetic field as well as its field and temperature sensitive magnetic phase transition. Additionally, the large value of $-\Delta S_{\mathrm{M}}^{\max }$ of $16.0 \mathrm{~J} \mathrm{~kg}^{-1} \mathrm{~K}^{-1}$ is reached under the field changes of $0-2 \mathrm{~T}$ with a quite wide temperature range for present $\mathrm{GdCoC}_{2}$, which is quite beneficial to application.

Franco et al. revealed a universal behavior of the field dependence of $\Delta S_{\mathrm{M}}$ for the materials with a second-order transition, i.e. in ref. 28 , the $\Delta S_{\mathrm{M}}$ versus $T$ curves under different magnetic fields can collapse into a universal curve; therefore the influence of different magnetic fields can be ignored. All the curves are normalized to their respective maximum value as $\Delta S_{\mathrm{M}} / \Delta S_{\mathrm{M}}^{\max }$ and the axis of temperature is rescaled to $\theta$ below and above $T_{\mathrm{C}}$,

$$
\theta=\left\{\begin{array}{c}
-\left(T-T_{\mathrm{C}}\right) /\left(T_{\mathrm{r} 1}-T_{\mathrm{C}}\right) ; \quad T \leq T_{\mathrm{C}}, \\
\left(T-T_{\mathrm{C}}\right) /\left(T_{\mathrm{r} 2}-T_{\mathrm{C}}\right) ; \quad T>T_{\mathrm{C}}
\end{array}\right.
$$

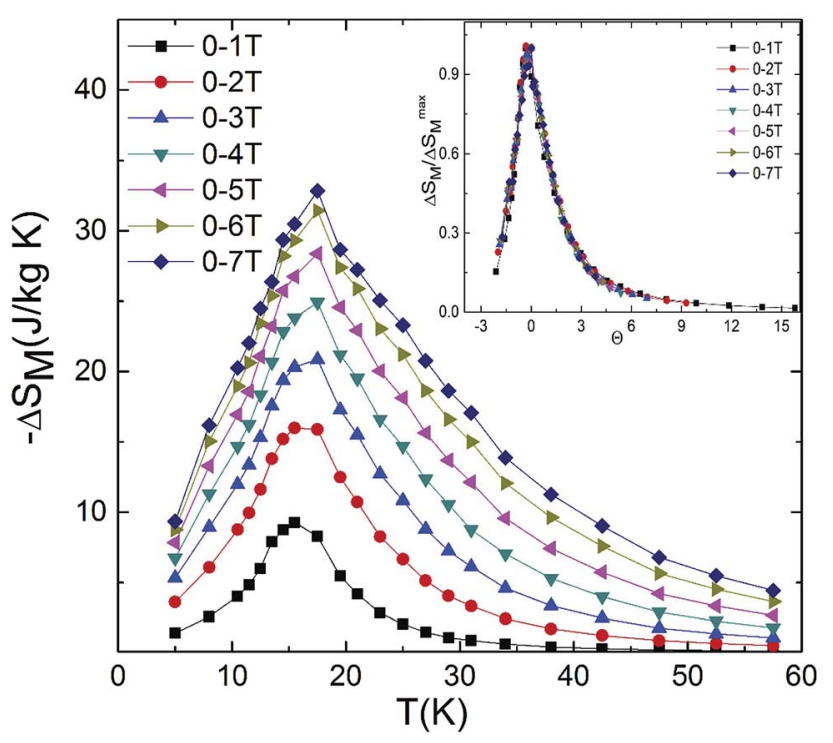

Fig. 4 Temperature-dependent magnetic entropy change $-\Delta S_{M}$ for $\mathrm{GdCOC}_{2}$ in various magnetic fields changes. The inset shows the universal curves.
Table 1 The transition temperature $\left(T_{M}\right),-\Delta S_{M}^{\max }$ and relative cooling

\begin{tabular}{|c|c|c|c|c|c|c|}
\hline & \multicolumn{3}{|c|}{$-\Delta S_{\mathrm{M}}^{\max }\left(\mathrm{J} \mathrm{kg}^{-1} \mathrm{~K}^{-1}\right)$} & \multicolumn{2}{|c|}{$\operatorname{RCP}\left(\mathrm{J} \mathrm{kg}^{-1}\right)$} & \multirow[b]{2}{*}{ Reference } \\
\hline & $T_{\mathrm{M}}(\mathrm{K})$ & $2 \mathrm{~T}$ & $5 \mathrm{~T}$ & $2 \mathrm{~T}$ & $5 \mathrm{~T}$ & \\
\hline $\mathrm{GdCoC}_{2}$ & 15 & 16.0 & 28.4 & 216 & 566 & Present \\
\hline $\mathrm{Eu}_{8} \mathrm{Ga}_{16} \mathrm{Ge}_{30}$ & 11 & 4.5 & 11.5 & 80 & 400 & 7 \\
\hline GdNiBC & 15 & 9.3 & 19.8 & 188 & 474 & 8 \\
\hline HoAgAl & 18 & 3.8 & 10.3 & 99 & 344 & 10 \\
\hline ErFeSi & 22 & 14.2 & 23.1 & $\sim 171$ & $\sim 486$ & 29 \\
\hline $\mathrm{GdCo}_{2} \mathrm{~B}_{2}$ & 25 & 9.3 & 17.1 & 167 & 462 & 14 \\
\hline $\mathrm{TbCoC}_{2}$ & 28 & 7 & 15.3 & $\sim 115$ & $\sim 375$ & 15 \\
\hline
\end{tabular}
power (RCP) under $2 \mathrm{~T}$ and $5 \mathrm{~T}$ for $\mathrm{GdCoC}_{2}$ and other materials with large

$T_{\mathrm{r} 1}$ and $T_{\mathrm{r} 2}$ are the temperatures of the two reference points of each curve that corresponds to $0.6-\Delta S_{\mathrm{M}}^{\max }$. The curves of normalized entropy change $\Delta S_{\mathrm{M}} / \Delta S_{\mathrm{M}}^{\max }$ versus the rescaled temperature $\theta$ under different magnetic fields are shown in the inset of Fig. 4. All the curves under different magnetic fields collapse onto a single line for $\mathrm{GdCoC}_{2}$, which further confirms that the present $\mathrm{GdCoC}_{2}$ undergoes a second order magnetic phase transition.

The relative cooling power (RCP) and refrigerant capacity (RC) are important factors to evaluate refrigeration materials. The values of RCP can be calculated as

$$
\mathrm{RCP}=-S_{\mathrm{M}}^{\max } \times \delta T_{\mathrm{FWHM}}
$$

$\delta T_{\mathrm{FWHM}}$ is the full width at half maximum and $-\Delta S_{\mathrm{M}}^{\max }$ is the maximum of magnetic entropy change, respectively. ${ }^{4}$ The values of RCP for $\mathrm{GdCoC}_{2}$ are $216,566,769 \mathrm{~J} \mathrm{~kg}^{-1}$ for $\Delta H=0-2,0-5$ and $0-7 \mathrm{~T}$, respectively. The values of $\mathrm{RC}$ can be calculated as

$$
\mathrm{RC}=\int_{T_{1}}^{T_{2}}\left|\Delta S_{\mathrm{M}}\right| \mathrm{d} T,
$$

$T_{1}$ and $T_{2}$ are the temperatures of half-maximum value of $-\Delta S_{\mathrm{M}}$ peak. ${ }^{4}$ The value of $\mathrm{RC}$ for $\mathrm{GdCoC}_{2}$ are equal to $160,369,514 \mathrm{~J}$ $\mathrm{kg}^{-1}$ for $\Delta H=0-2,0-5$ and $0-7 \mathrm{~T}$, respectively. For a comparison, the transition temperature $\left(T_{\mathrm{M}}\right)$, together with the values of $-\Delta S_{\mathrm{M}}^{\max }, \mathrm{RCP}$ and $\mathrm{RC}$ with the field change from 0 to $2 \mathrm{~T}$ and 5 $\mathrm{T}$ for $\mathrm{GdCoC}_{2}$ and some recently reported large/giant MCE materials with $T_{\mathrm{M}}$ around $15 \mathrm{~K}$ are shown in Table 1 . These values for $\mathrm{GdCoC}_{2}$ are obviously larger than most of those reported materials in the similar working temperature range.

\section{Conclusions}

In summary, a single phase $\mathrm{GdCoC}_{2}$ compound is synthesized, and its crystal structure, magnetic properties and magnetocaloric properties have been investigated. The compound undergoes a second-order paramagnetic to ferromagnetic (PMFM) transition at the Curie temperature $T_{\mathrm{C}} \sim 15 \mathrm{~K}$. Accompanied with the transition, giant reversible MCE is observed. The values of $-\Delta S_{\mathrm{M}}^{\max }$ for $\mathrm{GdCoC}_{2}$ reach $16.0,28.4$, and $32.9 \mathrm{~J} \mathrm{~kg}^{-1}$ $\mathrm{K}^{-1}$ for the field changes of 0-2 T, 0-5 T and 0-7 T, respectively. The corresponding values of RCP (RC) are 216 (160), 566 (369), 
and 769 (514) $\mathrm{J} \mathrm{kg}^{-1}$. For the reason that the compound has large MCE and small hysteresis in a wide temperature range, it could be considered as potential refrigerant material for low temperature magnetic refrigeration.

\section{Acknowledgements}

This work was supported by the National Natural Science Foundation of China (Grant No. 11374081) and the Fundamental Research Funds for the Central Universities (Grant No. N150905001 and N140901001).

\section{References}

1 E. Bruck, J. Phys. D: Appl. Phys., 2005, 38, R381.

2 K. A. Gschneidner, V. K. Pecharsky and A. O. Tsokol, Rep. Prog. Phys., 2005, 68, 1479.

3 B. G. Shen, J. R. Sun, F. X. Hu, H. W. Zhang and Z. H. Cheng, Adv. Mater., 2009, 21, 4545.

4 L. W. Li, Chin. Phys. B, 2016, 25, 037502.

5 A. Smith, C. R. H. Bahl, R. Bjørk, K. Engelbrecht, K. K. Nielsen and N. Pryds, Adv. Energy Mater., 2012, 2, 1288.

6 A. I. Vinokur and D. C. Fredrickson, Inorg. Chem., 2016, 55, 6148.

7 A. Chaturvedi, S. Stefanoski, M. H. Phan, G. S. Nolas and H. Srikanth, Appl. Phys. Lett., 2011, 99, 162513.

8 L. W. Li, M. Kadonaga, D. X. Huo, Z. H. Qian, T. Namiki and K. Nishimura, Appl. Phys. Lett., 2012, 101, 122401.

9 Y. Zhang, Y. Yang, X. Xu, L. Hou, Z. Ren, X. Li and G. Wilde, J. Phys. D: Appl. Phys., 2016, 49, 145002.

10 Y. Zhang, B. Yang and G. Wilde, J. Alloys Compd., 2015, 619, 12.

11 K. P. Shinde, S. H. Jang, J. W. Kim, D. S. Kim, M. Ranot and K. C. Chung, Dalton Trans., 2015, 20386.

12 T. Paramanik, T. Samanta, R. Ranganathan and I. Das, RSC $A d v ., 2015$, 5, 47860.

13 B. Sattibabu, A. K. Bhatnagar, K. Vinod, S. Rayaprol, A. Mani, V. Siruguri and D. Das, RSC Adv., 2016, 6, 48636.
14 L. W. Li, K. Nishimura and H. Yamane, Appl. Phys. Lett., 2009, 94, 102509.

15 B. Li, W. J. Hu, X. G. Liu and F. Yang, Appl. Phys. Lett., 2008, 92, 242508.

16 S. Yin, V. Sharma, A. McDannald, F. A. Reboredo and M. Jain, RSC Adv., 2016, 6, 9475.

17 Y. Zhang, L. Hou, Z. Ren, X. Li and G. Wilde, J. Alloys Compd., 2016, 565, 635.

18 B. Uthaman, K. S. Anand, R. K. Rajan, H. H. Kyaw, S. Thomas, S. Al-Harthi, K. G. Suresh and M. R. Varma, RSC Adv., 2015, 5, 86144.

19 Y. K. Zhang, X. Xu, Y. Yang, L. Hou, Z. Ren, X. Li and G. Wilde, J. Alloys Compd., 2016, 667, 130.

20 H. Amanai, H. Onodera, M. Ohashi, S. Matsuo, H. Yamauchi, Y. Yamaguchi and N. Sato, J. Magn. Magn. Mater., 1995, 148, 413.

21 H. Onodera, N. Uchida, M. Ohashi, H. Yamauchi, Y. Yamaguchi and N. Sato, J. Magn. Magn. Mater., 1994, 137, 35.

22 P. A. Kotsanidis, J. K. Yakinthos and W. Schafer, J. Alloys Compd., 1996, 242, 90.

23 W. Schafer, G. Will, P. A. Kotsanidis and J. K. Yakinthos, J. Magn. Magn. Mater., 1990, 88, 13.

24 W. Schafer, W. Kockelmann, G. Will, J. K. Yakinthos and P. A. Kotsanidis, J. Alloys Compd., 1997, 250, 565.

25 M. Reehuis, J. Rodriguez-Carvajal, M. E. Danebrock and W. Jeitschko, J. Magn. Magn. Mater., 1995, 151, 273.

26 W. Schafer, W. Kockelman, G. Will, P. A. Kotsanidis, J. K. Yakinthos and J. Linhart, J. Magn. Magn. Mater., 1994, 132, 243.

27 B. K. Banerjee, Phys. Lett., 1964, 12, 16.

28 V. Franco, A. Conde, V. Provenzano and R. D. Shull, J. Magn. Magn. Mater., 2010, 322, 218.

29 H. Zhang, B. G. Shen, Z. Y. Xu and J. Shen, Appl. Phys. Lett., 2013, 102, 092401. 